\title{
Achilles tendon reconstruction combining a modified Dresden technique and endoscopic flexor hallucis longus transfer
}

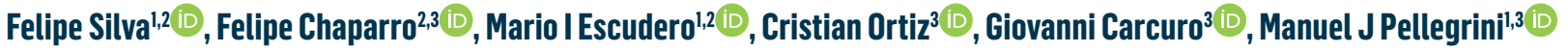 \\ 1. Hospital Clínico Universidad de Chile, Independencia, Región Metropolitana, Santiago, Chile. \\ 2. Hospital Clínico San Jose, Independencia, Región Metropolitana, Santiago, Chile. \\ 3. Universidad de los Andes, Las Condes, Region Metropolitana, Santiago, Chile.
}

\begin{abstract}
Objective: The purpose of this paper is to describe a minimally invasive chronic Achilles tendon rupture reconstruction combining a modified Dresden technique and endoscopic flexor hallucis longus (FHL) tendon transfer.

Methods: Our prospectively collected database was queried for patients presenting with chronic Achilles tendon rupture. Patients were included if they presented any of the following criteria: more than 65 years of age, history of previous DVT, active smoking habit and Diabetes. Pre and post-operative SF-36 and AOFAS hindfoot scores, complications, and patient satisfaction grades were recorded.

Results: Eight patients met the inclusion criteria; the median age was 49 years old (range 22 - 67 years). Two complications were registered (sural neuritis and minor wound dehiscence). Mean AOFAS score increased from 48 (range 40 - 63) to 91,6 (range 85 - 95). Regarding SF-36 score, the SFF-36 improved from 51,6 to 79,3 points and the SFM-36 enhance from 25 to 61,5 points. All patients evaluated their satisfaction regarding the performed procedure as satisfactory.
\end{abstract}

Conclusion: Chronic Achilles tendon rupture reconstruction combining a modified Dresden technique and endoscopic FHL transfer is an attractive option in high-risk patients, with favorable results at the short-term follow-up.

Level of Evidence IV; Therapeutic Study; Case Series.

Keywords: Achilles tendon/injuries; Achilles tendon/surgery; Tendon transfer; Reconstructive surgical procedures/methods; Endoscopy/methods.

\section{Introduction}

Chronic Achilles tendon ruptures (CATR) can produce elongation of the Gastrocnemius-Soleus complex, generating calf soreness, muscle cramping and/or functional disability ${ }^{(1)}$. Although several authors have tried to organize surgical treatment creating algorithms based on the tendon gap size, optimal surgical treatment remains to be elucidated(2).

Current treatment comprises some form of tendon reconstruction or tendons transfers in cases in which the muscular unit is scarred or presents severe fatty degeneration ${ }^{(1,2)}$. Although minimal invasive attempts to perform these operations have been advocated, most of these procedures are still performed through open surgery. In such a scenario, most case series report good functional outcomes after any of these procedures. Nevertheless, the patient faces a high risk of developing wound complications and infection, particularly those presenting risk factors such as old age, diabetes, smoking history and/or peripherical vascular disease.

Theoretically speaking, optimal surgical treatment must restore Gastrocnemius-Soleus complex length through a minimally invasive procedure, preserving muscular strength of the affected limb and diminishing complications risk.

The purpose of this study is to present our technique to address Chronic Achilles ruptures, combining a Dresden percutaneous technique, which is usually performed for acute ruptures, and an endoscopic FHL transfer.
Study performed at the Department of Orthopaedic Surgery, Hospital Clínico Universidad de Chile, Independencia, Región Metropolitana, Santiago, Chile.

Correspondence: Manuel J Pellegrini. 2501 Plaza Av., las Condes, Santiago, Chile, Zip Code: 7550000. E-mail: mpellegrini@clinicauandes.cl. Conflicts of interest: none. Source of funding: none. Date received: April 07, 2020. Date accepted: April 07, 2020. Online: April 30, 2020
Silva F, Chaparro F, Escudero MI, Carcuro G, Ortiz

C, Pellegrini MJ. Achilles tendon reconstruction

combining a modified Dresden technique and endoscopic Flexor hallucis longus transfer. J Foot Ankle. 2020;14(1):14-8. 


\section{Methods}

This study was approved by the Institutional Review Board.

Our prospectively collected database was queried for patients presenting with Achilles' tendon ruptures or re-ruptures with at least six weeks since the injury. Patients were included if they presented any of the following criteria: more than 65 years of age, history of previous DVT, active smoking habit, and diabetes. Patients with neurological disorders or not willing to participate were excluded from the study.

Pre and post-operative SF-36 and AOFAS hindfoot scores, complications, and patient satisfaction grades were recorded. Each patient assed their treatment satisfaction using a previously published scale as follows: Satisfied, satisfied with minor objections, satisfied with major objections, and unsatisfied $^{(3)}$.

\section{Surgical technique}

The procedure is performed under spinal anesthesia. The patient is placed prone in the operative table with a pneumatic thigh tourniquet at 250 The foot is placed at the edge of the table over a padded cushion that allows free movement of the ankle joint and to clear the contralateral limb from the operative area.

Traditional posteromedial and posterolateral arthroscopic portals are outlined and performed immediately adjacent to the Achilles' tendon. A $3.0 \mathrm{~mm}$ arthroscope and 4.0 shaver (Arthrex, Naples FL) are utilized to perform the posterior endoscopy. After identifying the $\mathrm{FHL}$ (Figure 1A), the tendon is freed for any surrounding tissue attachments, starting from the muscular belly to the posterior talar tubercles. This step will ensure that tendon retrieval will be safely performed. The deep fascia over the FHL muscular belly is opened with the shaver as well to enhance excursion.

In anticipation of FHL harvesting, the tendon is secured using a mini scorpion automatic suture passer (Arthrex, Naples FL) through the posteromedial portal. By folding the 2.0 Fiberwire suture (Arthrex, Naples FL) in half, a double suture is obtained. After piercing the $\mathrm{FHL}$ tendon with the automatic suture passer, sutures ends, and a loop are retrieved through the posteromedial portal. Both sutures end are introduced into the loop and pulled to deliver the loop into the posterior aspect of the ankle joint and grasp the tendon for manipulation. Thereafter, the ankle is positioned at maximum plantar flexion, and the FHL is pulled proximally through the sutures to harvest the tendon as distal as possible (Figure $1 \mathrm{C}$ and $\mathrm{C}$ ). After harvesting has been completed, the tendon is delivered through the posterolateral portal and prepared for transfer using 2-0 FiberLoop (Arthrex, Naples $F L$ ). This crucial step will prevent the tendon from being injured during tunnel preparation and prevent neurovascular damage at the medial side. FHL diameter is measured at this time to determine the size of the drilling guide, which will be of the same size of the harvested tendon (Figure 2A and B).
The dorsal aspect of the calcaneus needs to be debrided in anticipation of bone tunnel preparation and tendon transfer. Ideally speaking, the FHL needs to be transferred centered in the calcaneus and as closest as possible to the Achilles' tendon footprint, to emulate the vector pulling force of the native muscle.

Through the posteromedial portal, a guidewire is placed in the calcaneus under direct visualization and pulled out through the plantar skin in a dorsal-medial to a plantar-lateral direction (Figure $2 \mathrm{C}$ ). It seems wise to confirm the guide position under fluoroscopy to achieve an optimal position (Figure 2D). A $20 \mathrm{~mm}$ depth tunnel is performed over the guidewire, and the tendon transferred to this tunnel. Sutures at the $\mathrm{FHL}$ tendon are recovered at the plantar skin and with the ankle held in maximal plantarflexion, these sutures are maximally pulled and clamped in the desired position using a hemostatic clamp. During this maneuver, the surgeon must visualize the tendon advancing through the bone tunnel under direct arthroscopic visualization.

Approximately at 2 to $6 \mathrm{~cm}$ from the Achilles tendon insertion, the tendon gap is identified through palpation. A transverse incision is made over the gap, and blunt dissection is carefully performed though the paratenon, taking care not to damage the sural nerve. Fibrous (scar) tissue is identified and resected to restore the muscular unit length (Figure $3 \mathrm{~A}$ ).

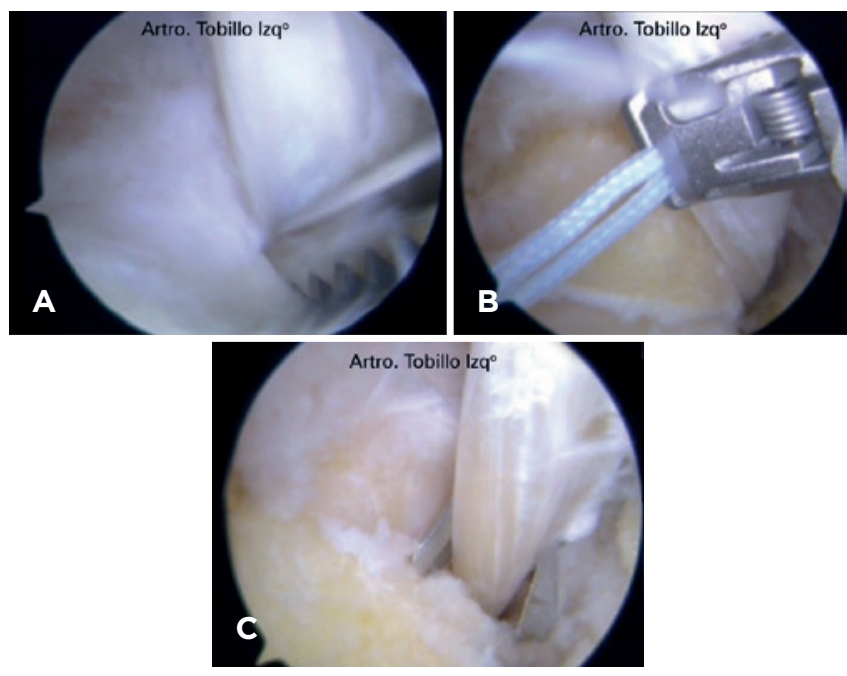

Figure 1. A. After FHL identification, the tendon is freed for any surrounding tissue attachments. B. The tendon is secured using a mini scorpion automatic suture passer (Arthrex, Naples FL) through the posteromedial portal. C. After positioning the ankle at maximum plantar flexion, and the $\mathrm{FHL}$ is pulled proximally through the sutures to harvest the tendon as distal as possible. 

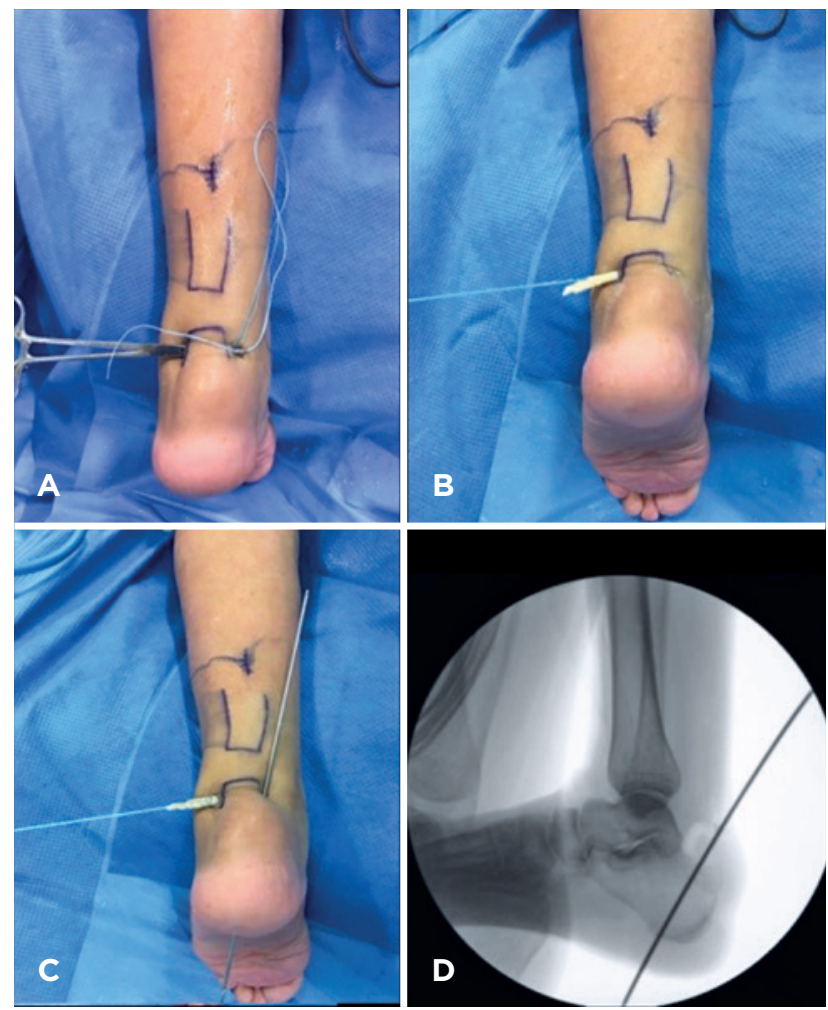

Figure 2. A. After harvesting has been completed, the tendon is delivered through the posterolateral portal. B. The tendon is prepared for transfer using 2-O FiberLoop (Arthrex, Naples FL). C. A guidewire is placed in the calcaneus under direct visualization and pulled out through the plantar skin in a dorsal-medial to a plantar-lateral direction. D. Guide position confirmation under fluoroscopy.

Using one Dresden instrument over the skin, a proximal incision is outlined and carried out to perform tenorrhaphy. Both Dresden instruments are delivered to the distal Achilles stump respecting the proximal paratenon, specifically between epiparatenon and subfascial plane, to avoid neurologic injuries. Two Fiberwire N 2 (Arthrex, Naples FL) sutures are percutaneously passed at the distal stump and recovered through the instruments at the proximal incision. Both sutures are then knotted to the proximal stump (Figure 3B and C). Once the Achilles tenorrhaphy has been performed, final $\mathrm{FHL}$ fixation is performed under arthroscopic visualization using a Biotenodesis screw one size under the diameter of the drilling guide (Arthrex, Naples FL) (Figure 3D).

\section{FHL fixation reinforcement}

In the last two cases, we have added tightrope fixation to the plantar side of the calcaneus to increase resistance for tendon pullout. In this situation, the tightrope button is loaded

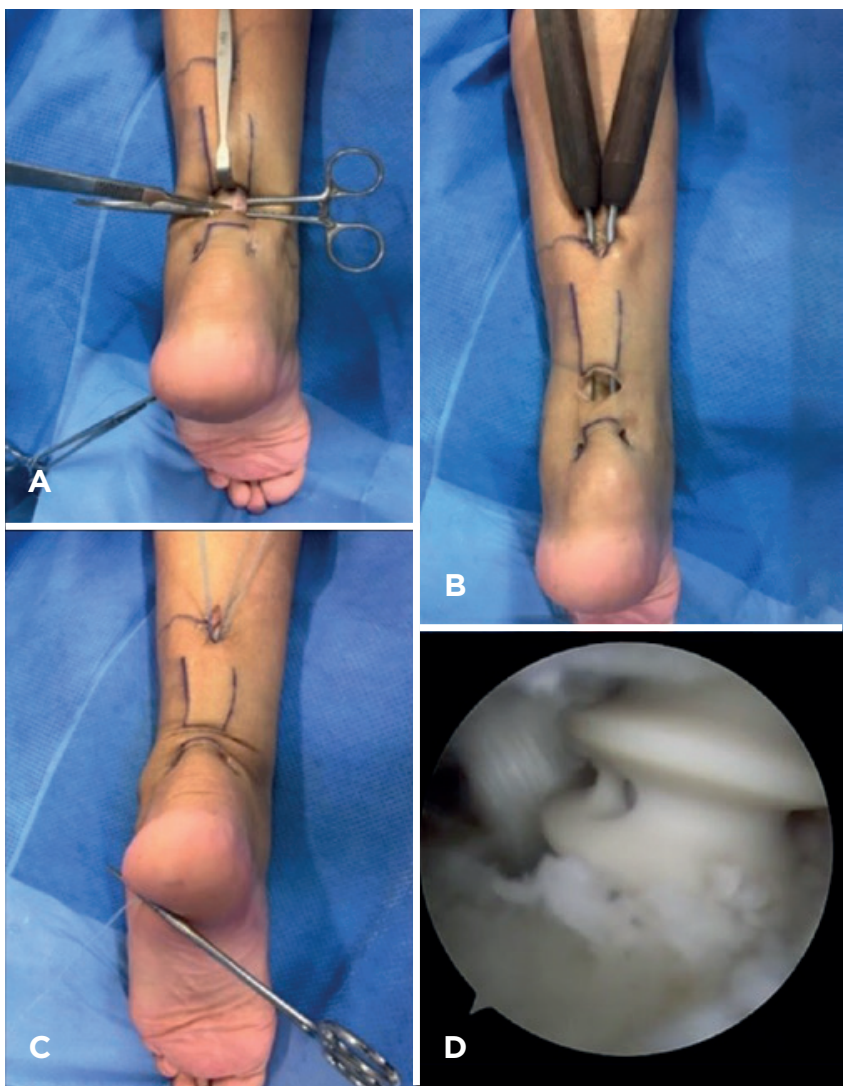

Figure 3. A. Fibrous (scar) tissue is identified and resected to restore the muscular unit length. B. Two Fiberwire N 2 (Arthrex, Naples FL) sutures are percutaneously passed at the distal stump and recovered through the instruments at the proximal incision. C. Both sutures are then knotted to the proximal stump. D. Final FHL fixation under arthroscopic visualization.

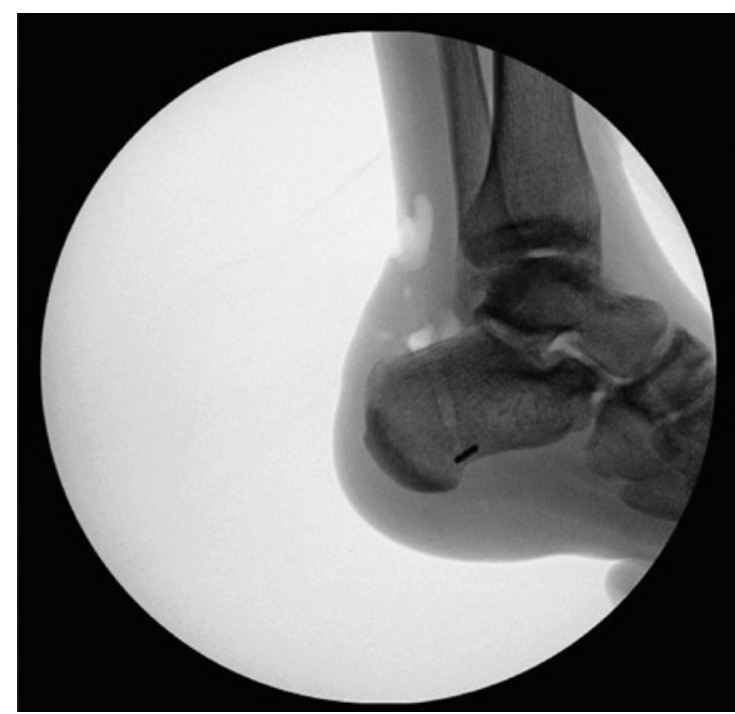

Figure 4. Checking tightrope fixation under fluoroscopy. 
with the FHL tendon sutures end and delivered through the bone tunnel. After checking under fluoroscopy that the tightrope is perpendicular to the tunnel, both sutures are cinched to deliver the tendon into the tunnel before biotenodesis screw fixation (Figure 4).

\section{Post-operative management}

The operated limb is immobilized in a cam-walker boot in equinus for three weeks, weight-bearing as tolerated with two crutches. Sutures are removed at 3 weeks, and immobilization maintained for three more weeks in neutral position. Physiotherapy is initiated after suture removal with exercises only in plantarflexion to neutral until six weeks postoperatively, when immobilization is discontinued. At post-operative week six, dorsiflexion exercises are encouraged and progressed according to patient tolerance.

\section{Results}

Eight patients met the inclusion criteria. Gender distribution was 6 male patients and 2 females. Median age was 49 years old (range 22 - 67 years). Mean time to surgery was 126 days (range from 60 to 300 days). Mean follow up was 27 months (range from 6 to 37 months). Mean AOFAS score increased from 48 (range 40 - 63) to 91,6 (range 85 - 95). Regarding SF-36 score, the SFF-36 component improved from 51,6 to 79,3 points and the SFM-36 component enhance from 25 to 61,5 points (Figure 5 and 6 ). All the patients evaluated the performed procedure as satisfactory. At final evaluation, almost all patients were able to perform single heel rise test. The only patient that was unable to elevate his heel was six months out from his surgery.

In terms of complications, one patient presented transient sural neuritis, which resolved spontaneously five months after surgery. Another patient presented a minor dehiscence of the transverse wound, which healed uneventfully without major intervention.

\section{Discussion}

Chronic Achilles tendon rupture is a debilitating condition due to Gastronecmius-Soleus complex dysfunction; this leads to gait impairment and functional disability affecting the patient's quality of life ${ }^{(4)}$. In this small case series, we demonstrate that our approach to chronic Achilles ruptures is a safe and effective procedure in the short term for patients over 65 years old, history of previous DVT, active smoking habit, and diabetes.

Several reconstruction techniques have been proposed(1). In general, defects greater than $6 \mathrm{cms}$. are best suited for allograft/autograft reconstruction or tendons transfer. Although good functional outcomes have been obtained with both approaches, they are not without complications, specially wound breakdown and infection (7 to $17 \%)^{(5,6)}$. Unfortunately, we have seen the same problems in our practice, especially in high-risk patients. In this scope, the FHL transfer is widely used in these situations. Unfortunately, any tendon transfer is underpowered to restore the Gastronecmius-Soleus complex muscular power ${ }^{(7)}$. The latter is supported by the fact that no muscle in the leg is strong enough to compare with the native Gastronecmius-Soleus muscular complex. Besides, transferring a tendon resembles the anatomic axis of muscular pulling, but it's not the same. In order to perform its function, the Achilles tendon depends on the tension applied through the muscular belly. If the tendon is elongated, the muscle will have to contract to a greater degree to produce the same action, leading to power loss and decreased endurance. For these reasons, we associated $\mathrm{FHL}$ transferring with a shortening of the elongated Achilles tendon ${ }^{(8,9)}$.

Alhaug et al. ${ }^{(10)}$ reported on 21 patients with open $\mathrm{FHL}$ transfer technique, with good functional outcomes, a median AOFAS score of 87.1 . However, a $52 \%$ complication rate, mostly by infection, makes this procedure less desirable. This rate of complications is similar with other manuscripts that have reported 17 to $25 \%$ wound complication rate with open tendon transfers ${ }^{(11)}$.

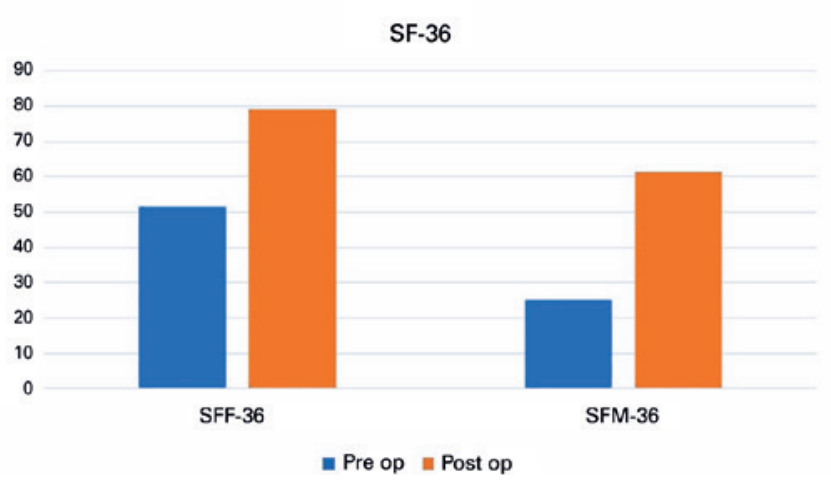

Figure 5. Pre and postoperative values for the physical (SFF-36) and mental (SFM-36) subscales of the SF 36 score.

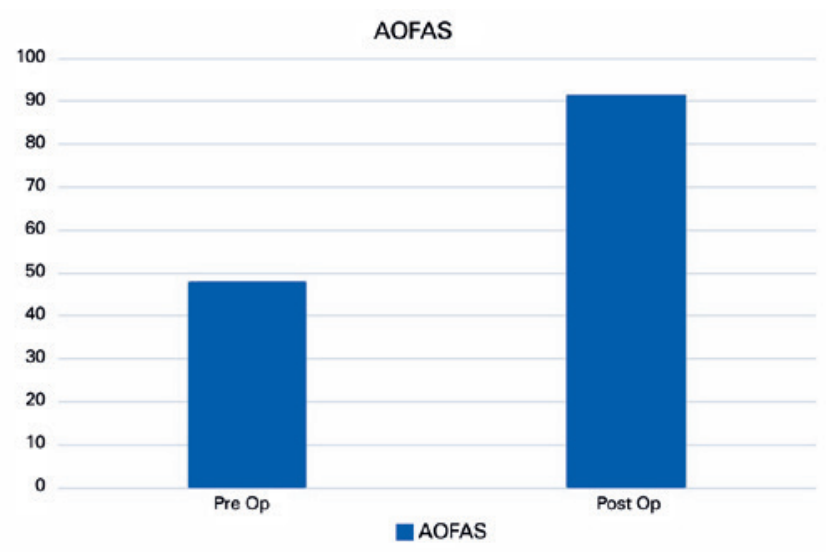

Figure 6. Pre and postoperative values for AOFAS Hindfoot and Ankle score. 
Conversion to endoscopic procedures have been advocated to diminish these complications, especially in high-risk patients. Vega et al. published on 22 patients, with no wound complications and all patients returning to their daily activities and improving their AOFAS hindfoot score from 54 to 91 . In a retrospective case series ${ }^{(12)}$. Husebye et al. ${ }^{(13)}$ describe endoscopic $\mathrm{FHL}$ transfers on 6 patients, all patients were followed for at least 12 months. At final follow up all patients described better function and increased plantar flexion power.

Our results compare favorably with these previously published studies. However, none of them but ours attempted to restore Gastrocnemius-Soleus complex length, which we believe is a simple procedure that can be achieved minimally invasive with a low complication rate. In summary, the endoscopic technique provides good functional results with a high level of patient satisfaction and low wound complication rate. However, technical complications can occur in relation to tendon harvesting or tunnel drilling.

Lately, we have augmented our FHL tendon transfer fixation in two cases. In terms of tendon fixation, a myriad of techniques has been described with more or less popularity ${ }^{(14)}$. Interference screw is an increasingly used technique because it decreases donor site morbidity, and less tendon length is needed for transfer, reducing the need of auxiliary incisions ${ }^{(14,15)}$. To the authors' knowledge, no clinical studies have described interference screw failure in FHL transfer. However, pull out cases of interference screws in biceps pathology have been reported(16). Therefore, we believe some patients could benefit from adding a tightrope button in the plantar aspect of the calcaneus, particularly in those with a poor bone quality or athletes requiring early sports reintegration.

\section{Conclusion}

This study is not without any limitations. The lack of a control group to compare this surgical approach, a limited and specific sample size (high-risk patients), and the short-term follow-up hinders our ability to draw definitive conclusions. However, we believe this technique is simple, reproducible, and safe to restore the gastrocnemius-soleus complex length and transferring the FHL tendon endoscopically in patients with chronic Achilles tendon ruptures.

Authors' contributions: Each author contributed individually and significantly to the development of this article: FSV *(https://orcid.org/O000-0003-39692916) wrote the article, interpreted the results of the study, participated in the review process approved the final version; FCR *(https://orcid.org/OOOO0001-6138-4384) wrote the article, participated in the review process, approved the final version; MEH *(https://orcid.org/O000-0002-8076-3360) wrote the article, participated in the review process, approved the final version; CC *(https://orcid.org/0000-0003-2574-9010) performed the surgeries and approved the final version; GC *(https://orcid.org/0000-0002-1993-6250) performed the surgeries and approved the final version; MJP *(https://orcid. org/O000-0002-2820-5337) performed the surgeries, wrote the article, approved the final version. ${ }^{*}$ ORCID (Open Researcher and Contributor ID) (iD).

\section{References}

1. Schweitzer KM Jr, Dekker TJ, Adams SB. Chronic Achilles ruptures: reconstructive options. J Am Acad Orthop Surg. 2018;26(21):753-63.

2. Chen $\mathrm{C}$, Hunt KJ. Open reconstructive strategies for chronic Achilles tendon ruptures. Foot Ankle Clin. 2019;24(3):425-37.

3. Pell G. Use and misuse of Likert scales. Med Educ. 2005;39(9):970; author reply 1 .

4. Hong CC, Lee WT, Murphy DP, Tan KJ. Anatomic basis for minimally invasive flexor hallucis longus transfer in chronic Achilles tendon rupture. J Foot Ankle Surg. 2018;57(5):938-41.

5. Acevedo JI, Weber KS, Eidelman DI. Technique tip: avoiding wound complications after neglected Achilles tendon repair using a tissue expansion technique. Foot Ankle Int. 2007;28(3):393-5.

6. Maffulli N, Longo UG, Gougoulias N, Denaro V. Ipsilateral free semitendinosus tendon graft transfer for reconstruction of chronic tears of the Achilles tendon. BMC Musculoskelet Disord. 2008;9:100.

7. Silver RL, de la Garza J, Rang M. The myth of muscle balance. A study of relative strengths and excursions of normal muscles about the foot and ankle. J Bone Joint Surg Br. 1985;67(3):432-7.

8. Koh D, Lim J, Chen JY, Singh IR, Koo K. Flexor hallucis longus transfer versus turndown flaps augmented with flexor hallucis longus transfer in the repair of chronic Achilles tendon rupture. Foot Ankle Surg. 2019;25(2):221-5.
9. Molloy A, Wood EV. Complications of the treatment of Achilles tendon ruptures. Foot Ankle Clin. 2009;14(4):745-59.

10. Alhaug OK, Berdal G, Husebye EE, Hvaal K. Flexor hallucis longus tendon transfer for chronic Achilles tendon rupture. A retrospective study. Foot Ankle Surg. 2019;25(5):630-5.

11. Syed TA, Perera A. Endoscopic management of chronic Achilles tendon rupture. Foot Ankle Clin. 2019;24(3):459-70.

12. Vega J, Vila J, Batista J, Malagelada F, Dalmau-Pastor M. Endoscopic flexor hallucis longus transfer for chronic noninsertional Achilles tendon rupture. Foot Ankle Int. 2018;39(12):1464-72.

13. Husebye EE, Molund M, Hvaal KH, Stodle AH. Endoscopic transfer of flexor hallucis longus tendon for chronic Achilles tendon rupture: technical aspects and short-time experiences. Foot Ankle Spec. 2018;11(5):461-6.

14. Lee J, Williams C, Lowrey C, Gould G, Markert R, Laughlin R. Flexor hallucis longus tendon transfer fixation. Foot Ankle Spec. 2017;10(1):31-6.

15. Khalid MA, Weiss WM, Iloanya M, Panchbhavi VK. Dual purpose use of flexor hallucis longus tendon for management of chronic Achilles tendon ruptures. Foot Ankle Spec. 2019;12(4):345-9.

16. Koch BS, Burks RT. Failure of biceps tenodesis with interference screw fixation. Arthroscopy. 2012;28(5):735-40. 